\title{
Exserohilum rostratum patógeno de Caryota mitis en Argentina
}

\author{
María A. Cúndom, Susana A. Gutiérrez ${ }^{1}$, Patricia Cejas ${ }^{1}$, María G. Cabrera ${ }^{1}$
}

${ }^{1}$ Fitopatología, Facultad de Ciencias Agrarias, Universidad Nacional del Nordeste, UNNE. Sargento Cabral 2131, 3400-Corrientes, Argentina. email: macundom@agr.unne.edu.ar

Data de chegada: 25/05/2005. Aceito para publicação em: 17/10/2005.

\section{RESUMEN}

Cúndom, M.A.; Gutiérrez, S.A.; Cejas, P.; Cabrera, M.G. Exserohilum rostratum patógeno de Caryota mitis en Argentina. Summa Phytopathologica, v.32, n.3, p.277-279, 2006.

Caryota mitis Loureiro (Arecaceae), es cultivada en Argentina como planta ornamental con fines comerciales. En el año 2003, muestras procedentes de la localidad de Castelli, provincia del Chaco, Argentina, presentaban atizonamiento de los ápices de las hojas, deteriorándolas considerablemente. Mediante métodos comunes de laboratorio se aisló un hongo Dematiaceae relacionado con los síntomas observados. Con base en las características culturales, morfométricas, pruebas de patogenicidad con resultados positivos, el agente causal fue identificado como Exserohilum rostratum (Drechsler) Leonard \& Suggs. Esta es la primera información de E. rostratum afectando a C. mitis en Argentina.

Palabras claves adicionales: etiología, palmera, mancha foliar.

\section{ABSTRACT}

Cúndom, M.A.; Gutiérrez, S.A.; Cejas, P.; Cabrera, M.G. Exserohilum rostratum on Caryota mitis in Argentina. Summa Phytopathologica, v.32, n.3, p.277-279, 2006.

Caryota mitis Loureiro (Arecaceae), is an ornamental plant of commercial interest in Argentina. In 2003, samples collected from Castelli, province of Chaco, Argentina, were affected by leaf blight and exhibited considerable damage. Using common laboratory techniques, a Dematiaceae associated with the

Additional keywords: etiology, palm, leaf spot. observable symptoms was isolated. Based on cultural studies, morphometric characteristics and pathogenicity test, the fungus was identified as Exserohilum rostratum (Drechsler) Leonard \& Suggs. This is the first report of E. rostratum affecting C. mitis in Argentina.

\section{RESUMO}

Cúndom, M.A.; Gutiérrez, S.A.; Cejas, P.; Cabrera, M.G. Exserohilum rostratum patógeno de Caryota mitis na Argentina. Summa Phytopathologica, v.32, n.3, p.277-279, 2006.

Caryota mitis Loureiro (Arecaceae), é cultivada na Argentina como planta ornamental com fins comerciais. No ano de 2003, amostras procedentes da localidade de Castelli, província do Chaco, Argentina, foram observadas apresentando necrose nas pontas das folhas, afetando a sua qualidade consideravelmente. Através de métodos comuns de laboratório isolou-se um fungo Dematiaceae associado com os sintomas observados. Com base nas características da cultura, as características morfométricas e os testes de patogenicidade com resultados positivos, o agente causal foi identificado como Exserohilum rostratum (Drechsler) Leonard \& Suggs. Este é o primeiro relato de E. rostratum afetando C. mitis na Argentina.

Palavras chave adicionais: etilogia, palmeira, mancha foliar.

La palmera cariota o cola de pescado, fishtail palm (Caryota mitis Lour.) (Arecaceae), es una especie muy cultivada en Argentina como planta ornamental.

En el año 2003, en la localidad de Castelli, Chaco, Argentina, se observaron plantas severamente afectadas por manchas foliares, irregulares, elípticas, en el sentido de las nervaduras, de tamaño variable, rodeadas de halo clorótico, formando grandes áreas necróticas. En análisis preliminares, se detectó la presencia de un hongo Dematiaceae de conidios castaño oliváceo, rectos a ligeramente curvos, con pseudoseptos.

En la revisión bibliográfica argentina no se encontraron antecedentes de la anormalidad observada. Chase (2) determinó por inoculaciones artificiales que Bipolaris, Exserohilum y Phaetrichoconis, son agentes causales de manchas foliares, en 
distintas especies de palmeras, entre ellas C. mitis; pudiendo ser lo suficientemente severas para reducir su valor comercial, debido a que la importancia de estas especies como ornamentales reside en la sanidad de sus hojas.

El presente trabajo se realizó con el objetivo de determinar la etiología de la enfermedad que afecta a $C$. mitis.

Los trabajos se llevaron a cabo en la Cátedra de Fitopatología de la Facultad de Ciencias Agrarias, UNNE. Se utilizaron métodos y técnicas de rutina en el estudio de enfermedades micológicas. Se estudió la evolución y secuencia de síntomas y signos en las muestras de hojas enfermas a simple vista y con microscopio estereoscópico (45 x).

Para favorecer la manifestación del signo, se prepararon cámaras húmedas que se colocaron en estufa $\left(26^{\circ} \mathrm{C}\right)$ en oscuridad y en condiciones de laboratorio $12 \mathrm{~h} \mathrm{luz}-12 \mathrm{~h}$ oscuridad.

Se realizaron siembras comunes del material enfermo tomando trozos de tejidos de $0,2 \mathrm{~cm}^{2}$ del borde entre tejido sano y enfermo, que se desinfectaron con hipoclorito de sodio al 2,5\%. Como medio de cultivo se empleó agar papa glucosado (APG) 1,5\%, $\mathrm{pH}$ 6,5, en placas de Petri, que luego de la siembra se incubaron en oscuridad durante siete días en estufa regulada a $26^{\circ} \mathrm{C}$.

Para el estudio de las características culturales y morfométricas, a partir de aislamientos monospóricos se realizaron siembras en APG, agar V8 (AV8), agar agua (AA), y en trocitos de hojas de sorgo en AA, incubadas en condiciones de laboratorio $12 \mathrm{~h} \mathrm{luz}$

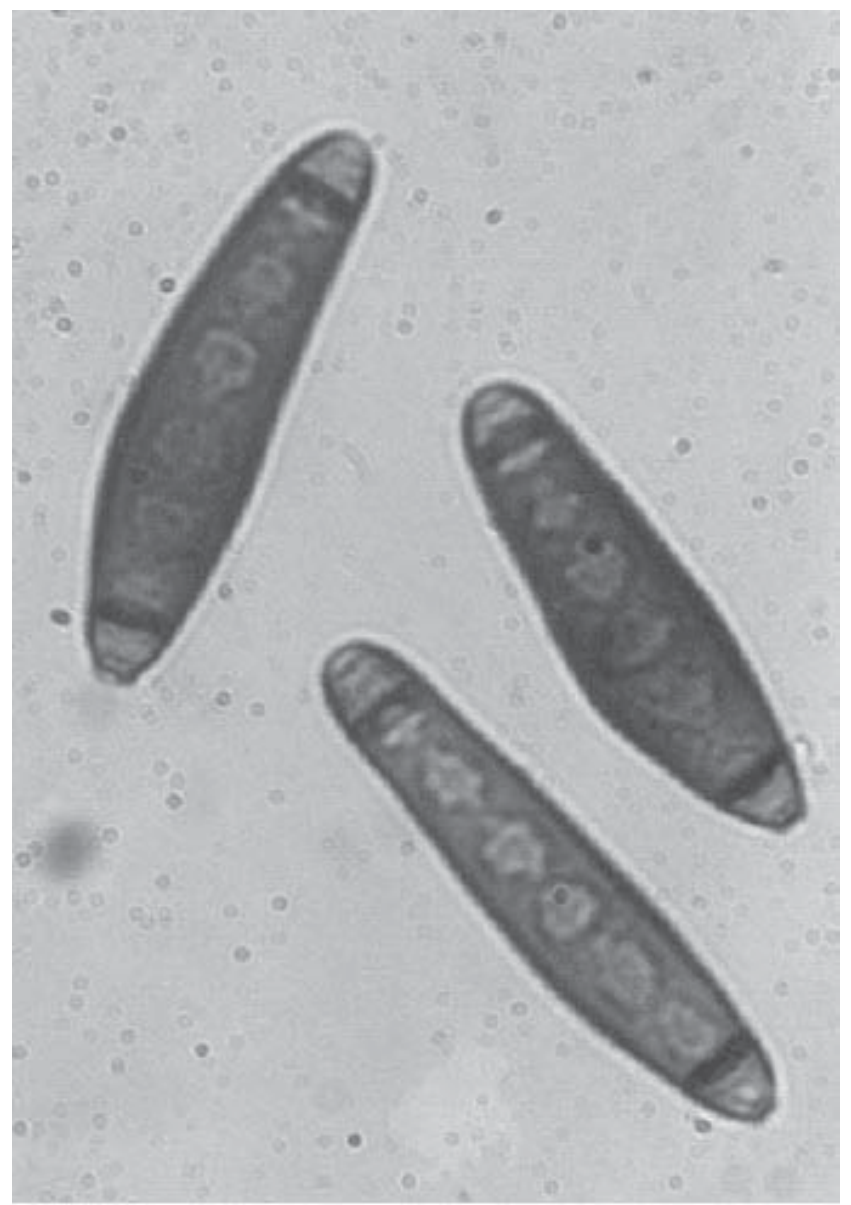

Figura 1. Conidios de Exserohilum rostratum agente causal de mancha foliar en Caryota mitis.
-12 h oscuridad.

Los conidios desarrollados en AV8 y en trocitos de sorgo en AA se midieron a los 14 días.

Para conocer el tipo de germinación, se realizaron observaciones de conidios "in vitro", preparando suspensiones de los mismos en agua destilada estéril.

La identificación del hongo se basó en los trabajos de Alcorn (1), Ellis (5), Leonard (6), Mathur \& Kongsdal (7) y Sivanesan (8).

Se realizaron pruebas de patogenicidad en plantas sanas de $C$. mitis criadas en macetas en invernáculo. Como inóculo se utilizó un aislamiento monospórico del hongo de 12 días, desarrollado en AV8, el cual fue depositado en ambas caras de hojas de cariota de dos formas: a) directamente sin heridas, y b) produciendo pequeñas heridas con aguja histológica. Luego las plantas se embolsaron y mantuvieron en condiciones de laboratorio durante 48 horas; posteriormente fueron llevadas a invernáculo. Se efectuaron reaislamientos a partir del material tratado. Los testigos se trataron de igual forma depositando trocitos de AV8 estéril sobre las hojas.

En APG, el hongo estudiado desarrolló micelio aéreo abundante, de crecimiento radial, al principio de color gris oliváceo, con suaves zonas concéntricas, luego oscureciéndose debido a la gran cantidad de conidios formados; el reverso de la colonia es gris muy oscuro. Microscópicamente $(400$ x) se observaron hifas

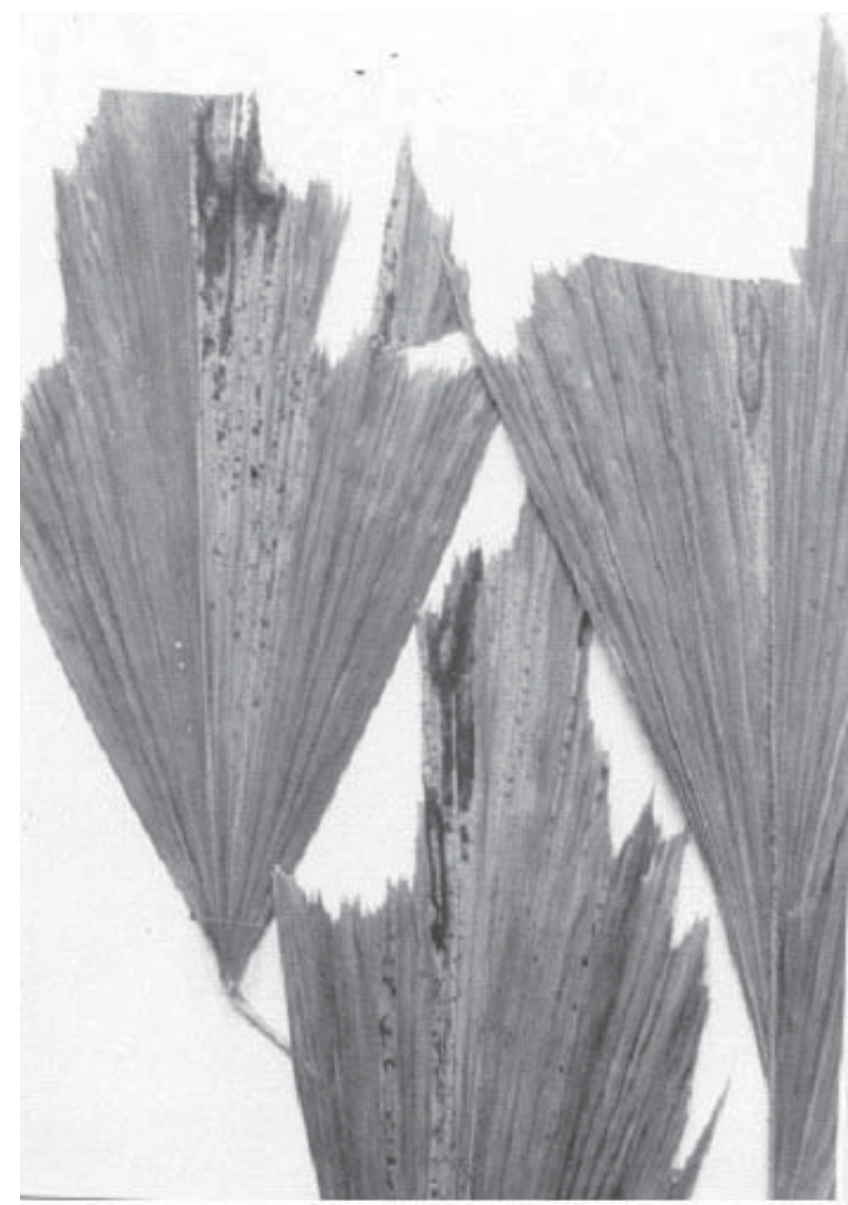

Figura 2. Hojas de Caryota mitis con manchas producidas Exserohilum rostratum. 
tabicadas, castaño más claro que los conidióforos. Conidióforos solitarios, a veces en pequeños grupos, rectos o flexuosos, tabicados, con numerosas cicatrices, de color castaño con el ápice más claro. Conidios rectos o ligeramente curvos, obclavados, no rostrados, castaño oliváceo, 3-10 pseudoseptos; las células de los extremos son más claras, separadas por septos más oscuros y anchos que los otros pseudoseptos, de 26-80 ìm de largo x 11-18 ìm de ancho, con hilo protuberante (Figura 1). La germinación de conidios in vitro es bipolar y el tubo germinativo adyacente al hilo, semiaxial. Las pruebas de patogenicidad en inoculaciones con heridas, dieron resultados positivos. Las primeras reacciones se observaron a los 18 días, produciendo manchas pequeñas de color castaño oscuro, irregulares, anfígenas. Al evolucionar, desarrollaron en lesiones más grandes, alargadas, elípticas, en el sentido de las nervaduras, de color castaño con centro más claro, rodeadas de halo clorótico (Figura 2). El hongo fue reaislado completándose los postulados de Koch.

Los antecedentes bibliográficos sobre manchas foliares causadas por hongos en $C$. mitis, son escasos $(2,3,4)$. Chase (2) inoculando varias especies de palmeras con Bipolaris, Exserohilum y Phaetrichoconis, determinó que la sintomatología no cambia sustancialmente sobre los hospedantes inoculados aunque el agente causal sea distinto en cada caso, lo cual dificulta su diagnóstico. En tanto Chase \& Broschat (3) informan que a pesar de la similitud de los síntomas, ellos son algo diferentes debido a la reacción propia de cada hospedante y al patógeno involucrado.

El seguimiento y la evolución de los síntomas en el substrato natural y en las pruebas de patogenicidad realizadas en C. mitis, concuerdan con la descripción de Chase (2) sobre este hospedante.

Los estudios realizados para la identificación del patógeno aislado, se basó en el trabajo de Alcorn (1), quien realizó un minucioso estudio para la diferenciación de los géneros
Drechslera, Bipolaris y Exserohilum, determinándose que el Dematiaceae causante de manchas foliares en la región, corresponde a Exserohilum.

Las características culturales y morfométricas se compararon con las descripciones dadas por Ellis (5), Leonard (6), Mathur \& Kongsdal (7) y Sivanesan (8), las que se ajustan a Exserohilum rostratum (Drechsler) Leonard \& Suggs $(=$ E. halodes (Drechsler) Leonard \& Suggs.

Esta es la primera referencia de E. rostratum afectando palmeras en Argentina.

\section{REFERENCIASBIBLIOGRAFICAS}

1. Alcorn, J.L. Generic concepts in Drechslera, Bipolaris and Exserohilum. Mycotaxon, Ithaca, v. 17, p. 1-86, 1983.

2. Chase, A.R. Deamatiaceous leaf spots of Crysalidocarpus lutescens and other palms in Florida. Plant Disease, St. Paul, v. 66, p. 697699, 1982.

3. Chase, A.R.; Broschat, T.K. Diseases and disorders of ornamental palms. 3 ed. St. Paul: APS Press, 1998. 56 p.

4. Coelho Netto, R. A.; Ferreira, F.A.; Assis, L. A.G. Cylindrocladium pteridis, agente causal de mancha foliar em Caryota mitis. Fitopatologia Brasileira, Brasília, v. 28, p. 569, 2003.

5. Ellis, M.B. Dematiaceous Hyphomycetes. Kew: Commonwealth Mycological Institute, 1971. 507 p.

6. Leonard, K.J. Synonymy of Exserohilum halodes with E. rostratum, and induction of the ascigerous state, Setosphaeria rostrata. Mycologia, Ithaca, v. 68, p. 402-411, 1976.

7. Mathur, S.B.; Kongsdal O. Common laboratory seed health testing methods for detecting fungi. Copenhagen: International Seed Testing Association (ISTA), 2003. 425 p.

8. Sivanesan, A. Graminicolous species of Bipolaris, Curvularia, Drechslera, Exserohilum and their teleomorphs. Kew: $\mathrm{CAB}$ International Mycological Institute, 1987. 261 p. (Mycological Paper, 158). 